\title{
Convergence Analysis of an Iterative Method for Nonlinear Partial Differential Equations
}

\author{
Hung-Yu Ke, Ren-Chuen Chen, and Chun-Hsien Li \\ Department of Mathematics, National Kaohsiung Normal University, Yanchao District, Kaohsiung City 82444, Taiwan \\ Correspondence should be addressed to Ren-Chuen Chen; rcchen@nknucc.nknu.edu.tw
}

Received 22 May 2013; Accepted 20 July 2013

Academic Editor: E. Karapinar

Copyright (c) 2013 Hung-Yu Ke et al. This is an open access article distributed under the Creative Commons Attribution License, which permits unrestricted use, distribution, and reproduction in any medium, provided the original work is properly cited.

\begin{abstract}
We will combine linear successive overrelaxation method with nonlinear monotone iterative scheme to obtain a new iterative method for solving nonlinear equations. The basic idea of this method joining traditional monotone iterative method (known as the method of lower and upper solutions) which depends essentially on the monotone parameter is that by introducing an acceleration parameter one can construct a sequence to accelerate the convergence. The resulting increase in the speed of convergence is very dramatic. Moreover, the sequence can accomplish monotonic convergence behavior in the iterative process when some suitable acceleration parameters are chosen. Under some suitable assumptions in aspect of the nonlinear function and the matrix norm generated from this method, we can prove the boundedness and convergence of the resulting sequences. Application of the iterative scheme is given to a logistic model problem in ecology, and numerical results for a test problem with known analytical solution are given to demonstrate the accuracy and efficiency of the present method.
\end{abstract}

\section{Introduction}

In terms of solving linear equations, we usually use two different iterative methods, namely, the Jacobi and GaussSeidel methods [1-3]. The monotone iterative (MI) schemes which combine linear iterative techniques, respectively, are presented and analyzed in [4-8] for solving nonlinear equations. The method of monotone iterations is a classical tool for the study of the existence of solutions of semilinear PDEs of certain types [9-12]. It is also useful for numerical solutions of these types of problems approximated, for instance, by the finite difference [5, 6, 13-15], finite element [16], or boundary element $[17,18]$ method. It is a constructive method that depends essentially on only one parameter, called the monotone parameter herein, which determines the convergent behavior of the iterative process. Besides, the block Picard, block Jacobi, and block Gauss-Seidel MI methods are also developed and compared the rates of convergence with the point MI schemes [6]. The block MI methods accelerate the rate of convergence more than the point MI methods. In particular, Ortega and Rheinboldt [19, page 456] mention an analysis of the Newton-SOR methods to research some properties of convergence for relaxation factor $0<\omega<1$. The MI methods have been widely used in the treatment of certain nonlinear parabolic and elliptic differential equations. For instance, in the study of certain subsonic flows and molecular interactions, the equation $\Delta u=u^{2}$ is of fundamental importance [18]. For parabolic problems with time delays we refer to [20]. In addition, we also utilize MI schemes to handle nonlinear problems on analysis of numerical results for semiconductor equations [21-23] and the Poisson Boltzmann equation [24].

Consider the nonlinear boundary-value problem:

$$
\begin{gathered}
-\left[\left(\mathrm{D}^{(1)} u_{x}\right)_{x}+\left(\mathrm{D}^{(2)} u_{y}\right)_{y}\right]=f(x, y, u), \quad \text { in } \Omega, \\
\alpha \frac{\partial u}{\partial v}+\beta u=g(x, y), \quad \text { on } \partial \Omega,
\end{gathered}
$$

in a two-dimensional domain $\Omega$ with boundary $\partial \Omega$, where $\partial u / \partial v$ is the outward normal derivative of $u$ on $\partial \Omega, \mathrm{D}^{(l)} \equiv$ $\mathrm{D}^{(l)}(x, y), l=1,2$, are positive functions on $\bar{\Omega} \equiv \Omega \cup \partial \Omega$, $\alpha \equiv \alpha(x, y)$ and $\beta \equiv \beta(x, y)$ are nonnegative functions on $\partial \Omega$ with $\alpha+\beta>0$, and $f$ and $g$ are given functions in their 
respective domains. For the nonlinear function $f$, we give two assumptions:

(i)

$$
f \text { is uniformly bounded for }-\infty<u<\infty \text {; }
$$

(ii) if $|u|,|v| \leq c$, then there exists a function $\mathscr{H}(c)$, such that for all $x, y \in \Omega$ we have

$$
|f(x, y, u)-f(x, y, v)| \leq \mathscr{H}(c) \cdot|u-v| .
$$

Applying the finite difference method to (1), we obtain a system of nonlinear algebraic equations in a compact form:

$$
A U=F(U)+G^{*} .
$$

Suppose that $A$ can be written in the splitting form $A=$ $\mathscr{D}-\mathscr{L}-\mathcal{U}$, where $\mathscr{D},-\mathscr{L}$, and $-\mathcal{U}$ are the diagonal, lower-offdiagonal, and upper-off-diagonal matrices of $A$, respectively. We consider that linear SOR method can be combined with nonlinear MI scheme to obtain a nonlinear SOR monotone iterative method for solving nonlinear equations which gives rise to the terminology "SORMI". The basic idea of this method joining MI method which depends essentially on the monotone parameter $\Gamma$ is that by introducing an acceleration parameter one can construct a sequence to accelerate the convergence. The algorithm is similar to the SOR method. Roughly speaking, given an initial vector $U^{(0)}$, the SORMI method generates a sequence of iterates $\left\{U^{(m)}\right\}, m=0,1, \ldots$, by solving the equation:

$$
\begin{aligned}
(\mathscr{D}+\Gamma-\omega \mathscr{L}) U^{(m+1)}= & {[(1-\omega)(\mathscr{D}+\Gamma)+\omega \mathscr{U}] U^{(m)} } \\
& +\omega\left[\Gamma U^{(m)}+F\left(U^{(m)}\right)+G^{*}\right],
\end{aligned}
$$

where $\omega$ is a relaxation factor. Under some suitable assumptions in aspect of the nonlinear function and the matrix norm generated from this method, we can prove the boundedness and convergence of the resulting sequences. Moreover, the sequences can accomplish monotonic convergence in the iterative process when some suitable relaxation factors are chosen.

The structure of the paper is as follows. In Section 2, we briefly make a description for discretization process to obtain algebraic equations for model (1) and state some properties of the matrix. Section 3 deals with the monotone parameter and constructs the SORMI scheme. We show the boundedness and convergence of the SORMI sequence in Sections 4 and 5. Moreover, we offer another proof for the convergence of the SORMI sequence in the case $0<\omega<1$. In Section 6 , we solve a one dimensional problem, and a logistic model in population growth problem and numerical results of the method are also given to verify the theoretical analysis. The final section is for some concluding remarks.

\section{A Finite Difference Discretization}

We discuss problem (1) in a rectangular domain $\Omega=\left(0, l_{1}\right) \times$ $\left(0, l_{2}\right)$. Let $h=l_{1} / n, k=l_{2} / m$, and let $x_{i}=i h, y_{j}=j k$ for $i=0,1,2, \ldots, n, j=0,1,2, \ldots, m$. The set of points $\left(x_{i}, y_{j}\right)$ in $\Omega$ and $\bar{\Omega} \equiv \Omega \cup \partial \Omega$ are defined, respectively, by $\Upsilon$ and $\bar{\Upsilon}$. When no confusion arises we write a point $\left(x_{i}, y_{j}\right)$ in $\bar{\Upsilon}$ by $(i, j) \in \bar{\Upsilon}$. Define

$$
\begin{gathered}
u_{i, j}=u\left(x_{i}, y_{j}\right), \\
f_{i, j}\left(u_{i, j}\right)=f\left(x_{i}, y_{j}, u\left(x_{i}, y_{j}\right)\right), \\
g_{i, j}=g\left(x_{i}, y_{j}\right) .
\end{gathered}
$$

The finite difference method for differential and boundary operators in (1) leads to a discrete system in the form

$$
\begin{aligned}
a_{i j} u_{i j} & -b_{i j} u_{i-1, j}-b_{i j}^{\prime} u_{i+1, j}-c_{i j} u_{i, j-1}-c_{i j}^{\prime} u_{i, j+1} \\
& =h k f_{i j}\left(u_{i j}\right)+g_{i j}^{*},
\end{aligned}
$$

for all $(i, j) \in \bar{\Upsilon}$, where the coefficients $a_{i j}, b_{i j}, b_{i j}^{\prime}, c_{i j}$, and $c_{i j}^{\prime}$ are associated with the diffusion coefficients $\mathrm{D}_{i j}^{(l)} \equiv \mathrm{D}^{(l)}\left(x_{i}, y_{j}\right)$, $l=1,2$, as well as the boundary coefficients $\alpha_{i j} \equiv \alpha\left(x_{i}, y_{j}\right)$ and $\beta_{i j} \equiv \beta\left(x_{i}, y_{j}\right), g_{i j}^{*}$ is associated with the boundary functions $g_{i j}$, and

$$
b_{0 j}=b_{n j}^{\prime}=c_{i 0}=c_{i m}^{\prime}=0
$$

for $i=0,1, \ldots, n, j=0,1, \ldots, m$,

$$
g_{i j}^{*}=0 \quad \text { for } i=1,2, \ldots, n-1, j=1,2, \ldots, m-1 .
$$

Typical choice of the coefficients in (5) for the interior mesh points is given by

$$
\begin{aligned}
& b_{i j}=\left(\frac{k}{h}\right) \mathrm{D}^{(1)}\left(x_{i}-\frac{h}{2}, y_{j}\right), \\
& b_{i j}^{\prime}=\left(\frac{k}{h}\right) \mathrm{D}^{(1)}\left(x_{i}+\frac{h}{2}, y_{j}\right), \\
& c_{i j}=\left(\frac{h}{k}\right) \mathrm{D}^{(2)}\left(x_{i}, y_{j}-\frac{k}{2}\right), \\
& c_{i j}^{\prime}=\left(\frac{h}{k}\right) \mathrm{D}^{(2)}\left(x_{i}, y_{j}+\frac{k}{2}\right), \\
& a_{i j}=b_{i j}+b_{i j}^{\prime}+c_{i j}+c_{i j}^{\prime}, \quad((i, j) \in \Upsilon)
\end{aligned}
$$

(e.g., see [25]). For the boundary points $\left(x_{i}, y_{j}\right)$, where $i=$ $0, n$ or $j=0, m$, the above coefficients are associated with the boundary coefficients $\alpha_{i j}$ and $\beta_{i j}$, and possess the property (for the case $\alpha>0$ ),

$$
a_{i j} \geq b_{i j}+b_{i j}^{\prime}+c_{i j}+c_{i j}^{\prime} \text { for } i=0, n \text { or } j=0, m \text {. }
$$

Furthermore, strict inequality in (8) holds for at least one $(i, j)$ when the boundary condition is not of pure Neumann condition. In either case, these coefficients satisfy the condition

$$
\begin{gathered}
b_{i j} \geq 0, \quad b_{i j}^{\prime} \geq 0, \quad b_{i j}+b_{i j}^{\prime}>0, \\
c_{i j} \geq 0, \quad c_{i j}^{\prime} \geq 0, \\
a_{i j} \geq b_{i j}+b_{i j}^{\prime}+c_{i j}+c_{i j}^{\prime}, \quad((i, j) \in \bar{\Upsilon}) .
\end{gathered}
$$


Condition (9) is our basic hypothesis for the boundedness and the convergence of the SORMI sequence. Now we rewrite system (5) in a compact form:

$$
A U=F(U)+G^{*} .
$$

Definition 1. A real $n \times n$ matrix $A=\left(a_{i j}\right)$ with $a_{i j} \leq 0$ for all $i \neq j$ and $a_{i i}>0$ for all $1 \leq i \leq n$ is an $\mathscr{M}$-matrix if $A$ is nonsingular, and $A^{-1} \geq 0$ [2].

Obviously, $A$ is a diagonally dominant with strict inequality for at least one $(i, j)$. Since the domain $\bar{Y}$ is connected, the above property implies that $A$ is nonsingular, and $A^{-1} \geq$ 0 . Hence, $A$ is an $\mathscr{M}$-matrix. This implies that for any nonnegative diagonal matrix $\mathbf{D},(A+\mathbf{D})^{-1}$ exists and is nonnegative.

Remark 2. Nonnegative matrices play a crucial role in the theory of matrices. They are important in the study of convergence of iterative methods and arise in many applications including economics, queuing theory, and chemical engineering. Let $A=\left(a_{i j}\right)$ and $B=\left(b_{i j}\right)$ be two real $n \times r$ matrices. Then, $A \geq B(A>B)$ if $a_{i j} \geq b_{i j}\left(>b_{i j}\right)$ for all $1 \leq i \leq n, 1 \leq j \leq r$. If $O$ is the null matrix and $A \geq O(>O)$, we say that $A$ is a nonnegative (positive) matrix. Since column vectors are $n \times 1$ matrices, we will use the terms nonnegative and positive vector throughout. A theorem which has important consequences on the analysis of iterative methods should be stated. Let $B$ be a nonnegative matrix. Then $\rho(B)<1$ if and only if $I-B$ is nonsingular and $(I-B)^{-1}$ is nonnegative, where $\rho(B)$ is the spectral radius of $B$.

Remark 3. In reality, the four conditions in the definition of $\mathscr{M}$-matrix are somewhat redundant, and equivalent conditions that are more rigorous will be (i) $a_{i j} \leq 0$ for all $i \neq j$, (ii) $A$ is nonsingular, and (iii) $A^{-1} \geq 0$. The condition, $a_{i i}>0$ for all $i$, is implied by the other three. Moreover, let $D$ be the diagonal of $A$, and $B \equiv I-D^{-1} A$. We can also obtain $\rho(B)<1$. A comparison theorem is as follows.

Let $A$ and $B$ be two $n \times n \mathscr{M}$-matrices, with $A \geq B$. Then we have $B^{-1} \geq A^{-1}$.

Remark 4. Let us look in more detail at the algebraic system (10) $[26,27]$. The connectedness assumption of $\Omega$ ensures that $A$ is irreducible. Condition (9) implies that $A$ is irreducibly diagonally dominant [28]. Let $A=\mathscr{D}-\mathscr{B}$, where $\mathscr{D}$ is the diagonal matrix of $A$. It can be shown that $0<\rho(\mathscr{B})<1$, using Perron-Frobenius theorem and the theory of regular splittings. A theorem states the following.

If $A=\left(a_{i j}\right)$ is a real $n \times n$ matrix with $a_{i j} \leq 0$ for all $i \neq j$, then the following are equivalent.

(i) $A$ is nonsingular, and $A^{-1}>0$.

(ii) The diagonal entries of $A$ are positive real numbers. $\mathscr{B}$ is nonnegative, irreducible, and convergent.

Thus, we know that $A$ is a diagonally dominant $\mathscr{M}$-matrix.

\section{The SORMI Method}

We now arrive to construct the SORMI sequence.

Definition 5. A vector $\widetilde{U} \equiv\left(\widetilde{u}_{00}, \widetilde{u}_{10}, \ldots, \widetilde{u}_{n 0}, \ldots, \widetilde{u}_{0 m}, \widetilde{u}_{1 m}\right.$, $\left.\ldots, \tilde{u}_{n m}\right)^{T}$ with $(n+1) \times(m+1)$ components is called an upper solution of (10) if

$$
A \widetilde{U} \geq F(\widetilde{U})+G^{*},
$$

and $\widehat{U} \equiv\left(\widehat{u}_{00}, \widehat{u}_{10}, \ldots, \widehat{u}_{n 0}, \ldots, \widehat{u}_{0 m}, \widehat{u}_{1 m}, \ldots, \widehat{u}_{n m}\right)^{T}$ is called a lower solution of (10) if

$$
A \widehat{U} \leq F(\widehat{U})+G^{*} .
$$

We say that $\widetilde{U}$ and $\widehat{U}$ are ordered if $\widetilde{U} \geq \widehat{U}$. Given any ordered upper and lower solutions $\widetilde{U}, \widehat{U}$, we set

$$
\langle\widehat{U}, \widetilde{U}\rangle \equiv\{U: \widehat{U} \leq U \leq \widetilde{U}\} .
$$

Define

$$
\begin{gathered}
\gamma_{i j} \equiv \max \left\{-\frac{\partial f_{i j}}{\partial u}\left(u_{i j}\right): \widehat{u}_{i j} \leq u_{i j} \leq \widetilde{u}_{i j}\right\}, \\
\gamma_{i j}^{+} \equiv \max \left\{0, \gamma_{i j}\right\}, \\
\Gamma \equiv h k \cdot \operatorname{diag}\left(\bar{\gamma}_{00}, \bar{\gamma}_{10}, \ldots, \bar{\gamma}_{n 0}, \bar{\gamma}_{01},\right. \\
\left.\bar{\gamma}_{11}, \ldots, \bar{\gamma}_{n 1}, \ldots, \bar{\gamma}_{0 m}, \bar{\gamma}_{1 m}, \ldots, \bar{\gamma}_{n m}\right),
\end{gathered}
$$

where $\bar{\gamma}_{i j}$ is any nonnegative scalar satisfying $\bar{\gamma}_{i j} \geq \gamma_{i j}^{+}$, and $\widetilde{u}_{i j}$ and $\widehat{u}_{i j}$ are the components of $\widetilde{U}$ and $\widehat{U}$, respectively. Then problem (10) is equivalent to

$$
(A+\Gamma) U=\Gamma U+F(U)+G^{*} .
$$

Suppose that $A$ can be written in the splitting form $A=$ $\mathscr{D}-\mathscr{L}-\mathscr{U}$, where $\mathscr{D},-\mathscr{L}$, and $-\mathscr{U}$ are the diagonal, lower-offdiagonal, and upper-off-diagonal matrices of $A$, respectively. The elements of $\mathscr{D}$ are positive, and those of $\mathscr{L}$ and $\mathscr{U}$ are nonnegative. Given an initial iterate vector $U^{(0)}$, the SOR method for solving the linear system $A U=b$ is

$$
(\mathscr{D}-\omega \mathscr{L}) U_{L}^{(m+1)}=[(1-\omega) \mathscr{D}+\omega \mathscr{U}] U_{L}^{(m)}+\omega b,
$$

where $\omega$ is a relaxation factor, and $U_{L}^{(0)}=U^{(0)}$. Moreover, the Gauss-Seidel MI method for solving the nonlinear system (15) is defined by

$$
(\mathscr{D}+\Gamma-\mathscr{L}) U_{G S}^{(m+1)}=(\Gamma+\mathscr{U}) U_{G S}^{(m)}+F\left(U_{G S}^{(m)}\right)+G^{*} .
$$

Thus, we define the SORMI method for solving the nonlinear system (15) by

$$
\begin{aligned}
(\mathscr{D}+\Gamma-\omega \mathscr{L}) U_{s}^{(m+1)}= & {[(1-\omega)(\mathscr{D}+\Gamma)+\omega \mathscr{U}] U_{s}^{(m)} } \\
& +\omega\left[\Gamma U_{s}^{(m)}+F\left(U_{s}^{(m)}\right)+G^{*}\right] .
\end{aligned}
$$




\section{The Boundedness of the SORMI Sequences}

Before the convergence analysis of the method, we want to ask whether the SORMI sequences are bounded. Now we consider the property.

Lemma 6. Given a pair of upper and lower solutions $\widetilde{U}, \widehat{U}$ of (10), let $U, V$ be two vectors with $(n+1) \times(m+1)$ components, and $\widetilde{U} \geq U \geq V \geq \widehat{U}$. Then

$$
\Gamma U+F(U) \geq \Gamma V+F(V) .
$$

Proof. Let $u_{i j}$ and $v_{i j}$ be the components of $U$ and $V$, respectively. By the mean value theorem,

$$
-\left[\frac{f\left(x_{i}, y_{j}, u_{i j}\right)-f\left(x_{i}, y_{j}, v_{i j}\right)}{u_{i j}-v_{i j}}\right]=-\frac{\partial f_{i j}}{\partial u}\left(w_{i j}\right),
$$

where $w_{i j}$ lies between $v_{i j}$ and $u_{i j}$. From (14), we have $\bar{\gamma}_{i j} \geq \gamma_{i j}^{+}$. Hence

$$
\begin{gathered}
-h k\left[\frac{f\left(x_{i}, y_{j}, u_{i j}\right)-f\left(x_{i}, y_{j}, v_{i j}\right)}{u_{i j}-v_{i j}}\right] \leq h k \gamma_{i j}^{+} \leq h k \bar{\gamma}_{i j}, \\
h k \bar{\gamma}_{i j} \cdot u_{i j}+h k f_{i j}\left(u_{i j}\right) \geq h k \bar{\gamma}_{i j} \cdot v_{i j}+h k f_{i j}\left(v_{i j}\right) .
\end{gathered}
$$

This completes the proof.

In [2, page 83], the theorem is stated as follows.

Theorem 7. If $A \geq O$ is an $n \times n$ matrix, then $\kappa>\rho(A)$ if and only if $\kappa I-A$ is nonsingular, and $(\kappa I-A)^{-1} \geq O$, where $\rho(A)$ is a spectral radius of $A$.

Hence, we quote the above theorem to obtain the following lemma.

Lemma 8. The matrix $(\mathscr{D}+\Gamma-\omega \mathscr{L})$ in (18) is nonsingular, and $(\mathscr{D}+\Gamma-\omega \mathscr{L})^{-1}$ is nonnegative for $0<\omega<2$.

Proof. From the splitting form of $A$ and (14), we have $(\mathscr{D}+\Gamma-$ $\omega \mathscr{L})=\left(a_{i j}+h k \bar{\gamma}_{i j}\right) I-\omega \mathscr{L}, i=0,1,2, \ldots, n, j=0,1,2, \ldots, m$, where $a_{i j}+h k \bar{\gamma}_{i j}>0$. Since $0<\omega<2, \omega \mathscr{L} \geq O$, and $\omega \mathscr{L}$ is a strictly lower triangle matrix. It follows that all eigenvalues of $\omega \mathscr{L}$ are zeros, and thus $\rho(\omega \mathscr{L})=0$. Hence, $a_{i j}+h k \bar{\gamma}_{i j}>\rho(\omega \mathscr{L})$. By Theorem 7, $(\mathscr{D}+\Gamma-\omega \mathscr{L})^{-1}=$ $\left[\left(a_{i j}+h k \bar{\gamma}_{i j}\right) I-\omega \mathscr{L}\right]^{-1} \geq O$.

Definition 9. Let the vector $U=\left(u_{00}, u_{10}, \ldots, u_{n 0}, \ldots\right.$, $\left.u_{0 m}, u_{1 m}, \ldots, u_{n m}\right)^{T}$. We define $|U|=\left(\left|u_{00}\right|,\left|u_{10}\right|, \ldots,\left|u_{n 0}\right|\right.$, $\left.\ldots,\left|u_{0 m}\right|,\left|u_{1 m}\right|, \ldots,\left|u_{n m}\right|\right)^{T}$.

Definition 10. Let $U=\left(u_{1}, u_{2}, \ldots, u_{n}\right)^{T} \in \Re^{n}$. The vector $2-$ norm is usually defined by $\|U\|=\left(\sum_{i=1}^{n} u_{i}^{2}\right)^{1 / 2}$.

Definition 11. Let $\|\cdot\|$ be a vector 2 -norm. The induced matrix 2-norm of an $n \times n$ matrix $A$ is defined by

$$
\|A\|=\sup _{U \neq 0} \frac{\|A U\|}{\|U\|} .
$$

Let $\left\{U_{s}^{(m)}\right\}_{m=0}^{\infty}$ be a sequence generated by the SORMI method with initial vector $U_{s}^{(0)} \in\langle\widehat{U}, \widetilde{U}\rangle$, where $\widetilde{U}, \widehat{U}$ are upper and lower solutions of (10), respectively. We have the following.

Lemma 12. $\|U\| \leq\||\widehat{U}|+|\widetilde{U}|\|$ for any $U \in\langle\widehat{U}, \widetilde{U}\rangle$. In fact, $\|U\|=\||U|\|$.

Proof. Let $U=\left(u_{00}, u_{10}, \ldots, u_{n 0}, \ldots, u_{0 m}, u_{1 m}, \ldots, u_{n m}\right)^{T}$, $\widehat{U}=\left(\widehat{u}_{00}, \widehat{u}_{10}, \ldots, \widehat{u}_{n 0}, \ldots, \widehat{u}_{0 m}, \widehat{u}_{1 m}, \ldots, \widehat{u}_{n m}\right)^{T}, \widetilde{U}=\left(\widetilde{u}_{00}, \widetilde{u}_{10}\right.$, $\left.\ldots, \widetilde{u}_{n 0}, \ldots, \widetilde{u}_{0 m}, \widetilde{u}_{1 m}, \ldots, \widetilde{u}_{n m}\right)^{T}$. Since $U \in\langle\widehat{U}, \widetilde{U}\rangle, \widehat{u}_{i j} \leq$ $u_{i j} \leq \widetilde{u}_{i j}$ for all $i=0,1,2, \ldots, n, j=0,1,2, \ldots, m$. Hence,

$$
\begin{aligned}
& u_{i j} \leq\left|u_{i j}\right| \leq \max \left\{\left|\widehat{u}_{i j}\right|,\left|\tilde{u}_{i j}\right|\right\} \leq\left|\widehat{u}_{i j}\right|+\left|\tilde{u}_{i j}\right|, \\
& \left(\sum_{i=0}^{n} \sum_{j=0}^{m} u_{i j}^{2}\right)^{1 / 2} \leq\left[\sum_{i=0}^{n} \sum_{j=0}^{m}\left(\left|\widehat{u}_{i j}\right|+\left|\widetilde{u}_{i j}\right|\right)^{2}\right]^{1 / 2} ;
\end{aligned}
$$

that is, $\|U\| \leq\||\widehat{U}|+|\widetilde{U}|\|$ for any $U \in\langle\widehat{U}, \widetilde{U}\rangle$.

To prove that the SORMI sequences are bounded, we must define several values about matrix and vector norms.

Notations and Assumptions. (a) Let $M_{1} \stackrel{\text { def }}{=}\|\widehat{U}\|+\|\widetilde{U}\|$. By Lemma 12,

$$
\left\|U_{s}^{(0)}\right\| \leq\||\widehat{U}|+|\widetilde{U}|\| \leq\|\widehat{U}\|+\|\widetilde{U}\|=M_{1} .
$$

(b) In (H1), we assume that $f_{i j}\left(u_{i j}\right)$ is uniformly bounded for $-\infty<u_{i j}<\infty$, and $g_{i j}^{*}$ is known boundary value; that is, there exists $\delta>0$ such that $\left|h k f_{i j}\left(u_{i j}\right)+g_{i j}^{*}\right| \leq \delta$ for $-\infty<$ $u_{i j}<\infty$, where $h$ and $k$ are mesh sizes. By Definition 10, (5) and (10), we have

$$
\left\|F(U)+G^{*}\right\| \leq\left[(n+1) \times(m+1) \times \delta^{2}\right]^{1 / 2}=\delta \sqrt{N} .
$$

(c) Define

$$
\begin{gathered}
M_{2}=\|E\|=\left\|(\mathscr{D}+\Gamma-\omega \mathscr{L})^{-1}\right\|, \\
\|B\|=\left\|(\mathscr{D}+\Gamma-\omega \mathscr{L})^{-1}[(1-\omega) \mathscr{D}+\Gamma+\omega \mathscr{U}]\right\|,
\end{gathered}
$$

and assume that

$$
\|B\|<1
$$

(d) Consider two vectors:

$$
\begin{aligned}
& U_{1} \stackrel{\text { def }}{=} \widehat{U}-\omega(\mathscr{D}+\Gamma-\omega \mathscr{L})^{-1}(\mathscr{D}+\Gamma)(\widetilde{U}-\widehat{U}), \\
& U_{2} \stackrel{\text { def }}{=} \widetilde{U}+\omega(\mathscr{D}+\Gamma-\omega \mathscr{L})^{-1}(\mathscr{D}+\Gamma)(\widetilde{U}-\widehat{U}) .
\end{aligned}
$$

Since $0<\omega<2,(\mathscr{D}+\Gamma-\omega \mathscr{L})^{-1} \geq O, \mathscr{D}+\Gamma \geq O$, and $\widetilde{U} \geq \widehat{U}$, we know that $\left[\omega(\mathscr{D}+\Gamma-\omega \mathscr{L})^{-1}(\mathscr{D}+\Gamma)(\widetilde{U}-\widehat{U})\right]$ is a nonnegative vector. It follows that $U_{1} \leq \widehat{U} \leq \widetilde{U} \leq U_{2}$. Let

$$
\begin{gathered}
\left\langle U_{1}, U_{2}\right\rangle \stackrel{\text { def }}{=}\left\{U: U_{1} \leq U \leq U_{2}\right\}, \\
M_{3} \stackrel{\text { def }}{=}\left\|\left|U_{1}\right|+\left|U_{2}\right|\right\| .
\end{gathered}
$$


Then

$$
\begin{aligned}
M_{3}= & \left\|\left|U_{1}\right|+\left|U_{2}\right|\right\| \leq\left\|U_{1}\right\|+\left\|U_{2}\right\| \\
= & \left\|\widehat{U}-\omega(\mathscr{D}+\Gamma-\omega \mathscr{L})^{-1}(\mathscr{D}+\Gamma)(\widetilde{U}-\widehat{U})\right\| \\
& +\left\|\widetilde{U}+\omega(\mathscr{D}+\Gamma-\omega \mathscr{L})^{-1}(\mathscr{D}+\Gamma)(\widetilde{U}-\widehat{U})\right\| \\
\leq & \|\widehat{U}\|+\omega\left\|(\mathscr{D}+\Gamma-\omega \mathscr{L})^{-1}(\mathscr{D}+\Gamma)\right\|(\|\widetilde{U}\|+\|\widetilde{U}\|) \\
& +\|\widetilde{U}\|+\omega\left\|(\mathscr{D}+\Gamma-\omega \mathscr{L})^{-1}(\mathscr{D}+\Gamma)\right\|(\|\widetilde{U}\|+\|\widetilde{U}\|) \\
< & \infty .
\end{aligned}
$$

(e) Define

$$
M_{4}=\max \left\{M_{1}, 2 \delta \sqrt{N} M_{2}\right\} .
$$

Theorem 13. Let $\widetilde{U}, \widehat{U}$ be a pair of ordered upper and lower solutions of (10), respectively, and let $\left\{U_{s}^{(m)}\right\}_{m=0}^{\infty}$ be a sequence generated by (18) with initial vector $U_{s}^{(0)} \in\langle\widehat{U}, \widetilde{U}\rangle$. Then, the iterative sequence $\left\{U_{s}^{(m)}\right\}_{m=0}^{\infty}$ is bounded for $0<\omega<2$.

Proof. By (28) and (30), we can choose a constant $M=$ $\max \left\{M_{3}, M_{4} /(1-\|B\|)\right\}$.

Consider two cases of the sequence $\left\{U_{s}^{(m)}\right\}$.

Case 1. Let $U_{s}^{(m-1)} \in\langle\widehat{U}, \widetilde{U}\rangle$. Then

$$
\begin{aligned}
(\mathscr{D}+\Gamma-\omega \mathscr{L}) U_{s}^{(m)}= & {[(1-\omega)(\mathscr{D}+\Gamma)+\omega \mathscr{U}] U_{s}^{(m-1)} } \\
& +\omega\left[\Gamma U_{s}^{(m-1)}+F\left(U_{s}^{(m-1)}\right)+G^{*}\right] \\
\leq & \mathscr{D} U_{s}^{(m-1)}+\Gamma U_{s}^{(m-1)}-\omega \mathscr{D} U_{s}^{(m-1)} \\
& -\omega \Gamma U_{s}^{(m-1)}+\omega \mathscr{U} U_{s}^{(m-1)} \\
& +\omega\left[\Gamma \widetilde{U}+F(\widetilde{U})+G^{*}\right] \\
\leq & \mathscr{D} U_{s}^{(m-1)}+\Gamma U_{s}^{(m-1)}-\omega \mathscr{D} U_{s}^{(m-1)} \\
& -\omega \Gamma U_{s}^{(m-1)}+\omega \mathscr{U} U_{s}^{(m-1)} \\
& +\omega[\Gamma \widetilde{U}+A \widetilde{U}] \\
\leq & \mathscr{D} \widetilde{U}+\Gamma \widetilde{U}-\omega \mathscr{D} \widehat{U}-\omega \Gamma \widehat{U}+\omega \mathscr{U} \widetilde{U} \\
& +\omega \Gamma \widetilde{U}+\omega \mathscr{D} \widetilde{U}-\omega \mathscr{L} \widetilde{U}-\omega \mathscr{U} \widetilde{U} \\
= & \omega(\mathscr{D}+\Gamma)(\widetilde{U}-\widehat{U}) \\
& +(\mathscr{D}+\Gamma-\omega \mathscr{L}) \widetilde{U} .
\end{aligned}
$$

By (27) and $(\mathscr{D}+\Gamma-\omega \mathscr{L})^{-1} \geq 0$, we obtain

$$
U_{s}^{(m)} \leq \widetilde{U}+\omega(\mathscr{D}+\Gamma-\omega \mathscr{L})^{-1}(\mathscr{D}+\Gamma)(\widetilde{U}-\widehat{U})=U_{2} .
$$

Similarly,

$$
(D+\Gamma-\omega \mathscr{L}) U_{s}^{(m)} \geq(\mathscr{D}+\Gamma-\omega \mathscr{L}) \widehat{U}-\omega(\mathscr{D}+\Gamma)(\widetilde{U}-\widehat{U}),
$$

and thus

$$
U_{s}^{(m)} \geq \widehat{U}-\omega(\mathscr{D}+\Gamma-\omega \mathscr{L})^{-1}(\mathscr{D}+\Gamma)(\widetilde{U}-\widehat{U})=U_{1} .
$$

So $U_{1} \leq U_{s}^{(m)} \leq U_{2}$. By Lemma 12 and (28), we obtain

$$
\left\|U_{s}^{(m)}\right\| \leq\left\|\left|U_{1}\right|+\left|U_{2}\right|\right\|=M_{3} \leq M .
$$

Case 2. Let $U_{s}^{(m-1)} \notin\langle\widehat{U}, \widetilde{U}\rangle$. Then

$$
\begin{aligned}
(\mathscr{D}+\Gamma-\omega \mathscr{L}) U_{s}^{(m)}= & {[(1-\omega)(\mathscr{D}+\Gamma)+\omega \mathcal{U}] U_{s}^{(m-1)} } \\
& +\omega\left[\Gamma U_{s}^{(m-1)}+F\left(U_{s}^{(m-1)}\right)+G^{*}\right] \\
= & {[(1-\omega) \mathscr{D}+\Gamma+\omega \mathcal{U}] U_{s}^{(m-1)} } \\
& +\omega\left[F\left(U_{s}^{(m-1)}\right)+G^{*}\right] .
\end{aligned}
$$

By (26), we have

$$
\begin{aligned}
U_{s}^{(m)}= & (\mathscr{D}+\Gamma-\omega \mathscr{L})^{-1}[(1-\omega) \mathscr{D}+\Gamma+\omega \mathscr{U}] U_{s}^{(m-1)} \\
& +\omega(\mathscr{D}+\Gamma-\omega \mathscr{L})^{-1}\left[F\left(U_{s}^{(m-1)}\right)+G^{*}\right] \\
= & B U_{s}^{(m-1)}+\omega E\left[F\left(U_{s}^{(m-1)}\right)+G^{*}\right] .
\end{aligned}
$$

Consider the iterative process. An induction argument gives

$$
\begin{aligned}
U_{s}^{(1)}= & B U_{s}^{(0)}+\omega E\left[F\left(U_{s}^{(0)}\right)+G^{*}\right], \\
U_{s}^{(2)}= & B U_{s}^{(1)}+\omega E\left[F\left(U_{s}^{(1)}\right)+G^{*}\right] \\
= & B\left\{B U_{s}^{(0)}+\omega E\left[F\left(U_{s}^{(0)}\right)+G^{*}\right]\right\} \\
& +\omega E\left[F\left(U_{s}^{(1)}\right)+G^{*}\right] \\
= & B^{2} U_{s}^{(0)}+\omega B E\left[F\left(U_{s}^{(0)}\right)+G^{*}\right] \\
& +\omega E\left[F\left(U_{s}^{(1)}\right)+G^{*}\right], \\
\vdots & \\
U_{s}^{(m)}= & B^{m} U_{s}^{(0)}+\omega B^{m-1} E\left[F\left(U_{s}^{(0)}\right)+G^{*}\right] \\
& +\omega B^{m-2} E\left[F\left(U_{s}^{(1)}\right)+G^{*}\right] \\
& +\cdots+\omega B E\left[F\left(U_{s}^{(m-2)}\right)+G^{*}\right] \\
& +\omega E\left[F\left(U_{s}^{(m-1)}\right)+G^{*}\right] .
\end{aligned}
$$


Hence, from Definition 11, (H3), (24), (25), (26), (30), and $0<$ $\omega<2$, we obtain

$$
\begin{aligned}
& \left\|U_{s}^{(1)}\right\| \leq\|B\| \cdot\left\|U_{s}^{(0)}\right\|+\omega \cdot\|E\| \cdot\left\|F\left(U_{s}^{(0)}\right)+G^{*}\right\| \\
& \leq M_{1}\|B\|+2 \delta \sqrt{N} M_{2} \\
& \leq M_{4}(\|B\|+1) \leq \frac{M_{4}}{1-\|B\|} \leq M, \\
& \left\|U_{s}^{(2)}\right\| \leq\|B\|^{2}\left\|U_{s}^{(0)}\right\|+\omega\|B\| \cdot\|E\| \cdot\left\|F\left(U_{s}^{(0)}\right)+G^{*}\right\| \\
& +\omega\|E\| \cdot\left\|F\left(U_{s}^{(1)}\right)+G^{*}\right\| \\
& \leq M_{1}\|B\|^{2}+2 \delta \sqrt{N} M_{2}\|B\|+2 \delta \sqrt{N} M_{2} \\
& \leq M_{4}\left(\|B\|^{2}+\|B\|+1\right) \leq \frac{M_{4}}{1-\|B\|} \leq M, \\
& \left\|U_{s}^{(m)}\right\| \leq\|B\|^{m}\left\|U_{s}^{(0)}\right\|+\omega\|B\|^{m-1}\|E\| \cdot\left\|F\left(U_{s}^{(0)}\right)+G^{*}\right\| \\
& +\omega\|B\|^{m-2}\|E\| \cdot\left\|F\left(U_{s}^{(1)}\right)+G^{*}\right\| \\
& +\cdots+\omega\|B\| \cdot\|E\| \cdot\left\|F\left(U_{s}^{(m-2)}\right)+G^{*}\right\| \\
& +\omega\|E\| \cdot\left\|F\left(U_{s}^{(m-1)}\right)+G^{*}\right\| \\
& \leq M_{4}\left(\|B\|^{m}+\cdots+\|B\|+1\right) \leq \frac{M_{4}}{1-\|B\|} \leq M .
\end{aligned}
$$

Thus, by Cases 1 and 2 , there exists $M>0$, such that $\left\|U_{s}^{(m)}\right\| \leq$ $M$ for all $m \in\{0\} \cup \mathcal{N}$, and the proof is completed.

\section{The Convergence of the SORMI Sequences}

In (H2), we assume that if $|u|,|v| \leq c$, then there is a function $\mathscr{H}(c)$, such that $|f(x, y, u)-f(x, y, v)| \leq \mathscr{H}(c) \cdot|u-v|$. From Theorem 13, we have shown the boundedness of the SORMI sequences; that is, there exists a constant $M$ such that $\left\|U_{s}^{(k)}\right\|<M=\max \left\{M_{3}, M_{4} /(1-\|B\|)\right\}$. Let $c_{k}$ satisfy $\max _{0 \leq i \leq m, 0 \leq j \leq n}\left|u_{i j}^{(k)}\right| \leq c_{k}$. So $0 \leq c_{k}<M$ for all $k$. Let $c_{0}=\sup _{k=1,2, \ldots}\left\{c_{k}\right\} \cdot c_{0}$ exists and $0 \leq c_{0}<M$. Hence, we can find the constant $\lambda \stackrel{\text { def }}{=} \mathscr{H}\left(c_{0}\right)$. So we have

$$
\begin{gathered}
\left|f\left(x_{i}, y_{j}, u_{i j}\right)-f\left(x_{i}, y_{j}, v_{i j}\right)\right| \leq \lambda\left|u_{i j}-v_{i j}\right|, \\
\left|h k f\left(x_{i}, y_{j}, u_{i j}\right)-h k f\left(x_{i}, y_{j}, v_{i j}\right)\right| \leq \lambda h k\left|u_{i j}-v_{i j}\right|, \\
|F(U)-F(V)| \leq \lambda h k|U-V| .
\end{gathered}
$$

Then we obtain the inequality:

$$
\begin{aligned}
\|F(U)-F(V)\| & =\||F(U)-F(V)|\| \\
& \leq \lambda h k\||U-V|\|=\lambda h k\|U-V\| .
\end{aligned}
$$

Theorem 14. Let $\widetilde{U}, \widehat{U}$ be a pair of ordered upper and lower solutions of (10), respectively, and let $\left\{U_{s}^{(m)}\right\}_{m=0}^{\infty}$ be a sequence generated by the SORMI method with initial vector $U_{s}^{(0)} \in$ $\langle\widehat{U}, \widetilde{U}\rangle$. Suppose that

$$
\eta \stackrel{\text { def }}{=}\|B\|+\omega \lambda h k\|E\|<1, \quad 0<\omega<2,
$$

where $B$ and $E$ are defined by (26). Then the sequence $\left\{U_{s}^{(m)}\right\}_{m=0}^{\infty}$ is convergent for $0<\omega<2$.

Proof. By Theorem 13, $\left\{U_{s}^{(m)}\right\}_{m=0}^{\infty}$ is bounded for $0<\omega<2$. Hence, $\exists M>0$, such that $\left\|U_{s}^{(m)}\right\| \leq M$ for all $m \in\{0\} \cup \mathcal{N}$. Since $\eta=\|B\|+\omega \lambda h k\|E\|<1$, and $0<\omega<2$, then, for every $\varepsilon>0, \exists N \in \mathcal{N}$, such that $\eta^{n}<\varepsilon / 2 M$ for all $n \geq N$. Let $k \geq m \geq N$. By (37), we have

$$
\begin{aligned}
U_{s}^{(k)}-U_{s}^{(m)}= & \left\{B U_{s}^{(k-1)}+\omega E\left[F\left(U_{s}^{(k-1)}\right)+G^{*}\right]\right\} \\
& -\left\{B U_{s}^{(m-1)}+\omega E\left[F\left(U_{s}^{(m-1)}\right)+G^{*}\right]\right\} \\
= & B\left(U_{s}^{(k-1)}-U_{s}^{(m-1)}\right) \\
& +\omega E\left[F\left(U_{s}^{(k-1)}\right)-F\left(U_{s}^{(m-1)}\right)\right] .
\end{aligned}
$$

Hence, from $0<\omega<2$ and (41), we obtain

$$
\begin{aligned}
\left\|U_{s}^{(k)}-U_{s}^{(m)}\right\|= & \| B\left(U_{s}^{(k-1)}-U_{s}^{(m-1)}\right) \\
& \quad+\omega E\left[F\left(U_{s}^{(k-1)}\right)-F\left(U_{s}^{(m-1)}\right)\right] \| \\
\leq & \|B\| \cdot\left\|U_{s}^{(k-1)}-U_{s}^{(m-1)}\right\| \\
& +\omega\|E\| \cdot\left\|F\left(U_{s}^{(k-1)}\right)-F\left(U_{s}^{(m-1)}\right)\right\| \\
\leq & \|B\| \cdot\left\|U_{s}^{(k-1)}-U_{s}^{(m-1)}\right\| \\
& +\omega \lambda h k\|E\| \cdot\left\|U_{s}^{(k-1)}-U_{s}^{(m-1)}\right\| \\
= & (\|B\|+\omega \lambda h k\|E\|) \cdot\left\|U_{s}^{(k-1)}-U_{s}^{(m-1)}\right\| \\
= & \eta\left\|U_{s}^{(k-1)}-U_{s}^{(m-1)}\right\| .
\end{aligned}
$$

Inductively we have

$$
\left\|U_{s}^{(k)}-U_{s}^{(m)}\right\| \leq \eta^{m}\left\|U_{s}^{(k-m)}-U_{s}^{(0)}\right\| .
$$

Hence,

$$
\begin{aligned}
\left\|U_{s}^{(k)}-U_{s}^{(m)}\right\| & \leq \eta^{m}\left\|U_{s}^{(k-m)}-U_{s}^{(0)}\right\| \\
& \leq \eta^{m}\left(\left\|U_{s}^{(k-m)}\right\|+\left\|U_{s}^{(0)}\right\|\right) \\
& \leq \eta^{m} \times 2 M<\frac{\varepsilon}{2 M} \times 2 M=\varepsilon .
\end{aligned}
$$

We have proved that $\left\{U_{s}^{(m)}\right\}_{m=0}^{\infty}$ is a Cauchy sequence for $0<$ $\omega<2$. It implies that $\left\{U_{s}^{(m)}\right\}_{m=0}^{\infty}$ is convergent for $0<\omega<$ 2. 
Furthermore, we provide another proof about the convergence of the SORMI sequences for $0<\omega<1$ without the assumptions (H3) and (H4). Denote the sequence by $\left\{\bar{U}_{s}^{(m)}\right\}_{m=0}^{\infty}$ when $U^{(0)}=\widetilde{U}$ and by $\left\{\underline{U}_{s}^{(m)}\right\}_{m=0}^{\infty}$ when $U^{(0)}=\widehat{U}$, and refer to them as the maximal and minimal sequences, respectively. The following theorem gives some monotone property of these sequences.

Theorem 15. The maximal and minimal sequences $\left\{\bar{U}_{s}^{(m)}\right\}_{m=0}^{\infty}$ and $\left\{\underline{U}_{s}^{(m)}\right\}_{m=0}^{\infty}$ given by (18) with $\widetilde{U}=\bar{U}_{s}^{(0)}$ and $\widehat{U}=\underline{U}_{s}^{(0)}$ possess the monotone property

$$
\widehat{U} \leq \underline{U}_{s}^{(m)} \leq \underline{U}_{s}^{(m+1)} \leq \bar{U}_{s}^{(m+1)} \leq \bar{U}_{s}^{(m)} \leq \widetilde{U}, \quad m=1,2, \ldots .
$$

Moreover for each $m, \bar{U}_{s}^{(m)}$ and $\underline{U}_{s}^{(m)}$ are ordered upper and lower solutions.

Proof. We will use induction to complete the proof of monotone property. First, let $W^{(0)}=\bar{U}_{s}^{(0)}-\bar{U}_{s}^{(1)}=\widetilde{U}-\bar{U}_{s}^{(1)}$. From (11), (18), and $0<\omega<1$,

$$
\begin{aligned}
(\mathscr{D}+\Gamma-\omega \mathscr{L}) W^{(0)}= & (\mathscr{D}+\Gamma-\omega \mathscr{L}) \widetilde{U}-(\mathscr{D}+\Gamma-\omega \mathscr{L}) \bar{U}_{s}^{(1)} \\
= & (\mathscr{D}+\Gamma-\omega \mathscr{L}) \widetilde{U} \\
& -\left\{[(1-\omega)(\mathscr{D}+\Gamma)+\omega \mathscr{U}] \bar{U}_{s}^{(0)}\right. \\
& \left.\quad+\omega\left[\Gamma \bar{U}_{s}^{(0)}+F\left(\bar{U}_{s}^{(0)}\right)+G^{*}\right]\right\} \\
= & \omega(\mathscr{D}-\mathscr{L}-\mathscr{U}) \widetilde{U}-\omega\left[F(\widetilde{U})+G^{*}\right] \\
= & \omega\left\{A \widetilde{U}-\left[F(\widetilde{U})+G^{*}\right]\right\} \geq 0 .
\end{aligned}
$$

By Lemma 8, we obtain $W^{(0)} \geq 0$. This leads to $\bar{U}_{s}^{(1)} \leq \bar{U}_{s}^{(0)}$. Similarly let $V^{(0)}=\underline{U}_{s}^{(0)}-\underline{U}_{s}^{(1)}=\widehat{U}-\underline{U}_{s}^{(1)}$, and use (12) to obtain

$$
(\mathscr{D}+\Gamma-\omega \mathscr{L}) V^{(0)}=\omega\left\{A \widehat{U}-\left[F(\widehat{U})+G^{*}\right]\right\} \leq 0 .
$$

Since $(\mathscr{D}+\Gamma-\omega \mathscr{L})^{-1} \geq 0$, it implies that $\underline{U}_{s}^{(1)} \geq \underline{U}_{s}^{(0)}$. Secondly, let $W^{(1)}=\bar{U}_{s}^{(1)}-\underline{U}_{s}^{(1)}$. By $(18)$,

$$
\begin{aligned}
(\mathscr{D}+\Gamma-\omega \mathscr{L}) W^{(1)}= & (\mathscr{D}+\Gamma-\omega \mathscr{L}) \bar{U}_{s}^{(1)} \\
& -(\mathscr{D}+\Gamma-\omega \mathscr{L}) \underline{U}_{s}^{(1)} \\
= & \left\{[(1-\omega)(\mathscr{D}+\Gamma)+\omega \mathscr{U}] \bar{U}_{s}^{(0)}\right. \\
& \left.+\omega\left[\Gamma \bar{U}_{s}^{(0)}+F\left(\bar{U}_{s}^{(0)}\right)+G^{*}\right]\right\} \\
& -\left\{[(1-\omega)(\mathscr{D}+\Gamma)+\omega \mathscr{U}] \underline{U}_{s}^{(0)}\right. \\
& \left.+\omega\left[\Gamma \underline{U}_{s}^{(0)}+F\left(\underline{U}_{s}^{(0)}\right)+G^{*}\right]\right\}
\end{aligned}
$$

$$
\begin{gathered}
=\{(1-\omega)(\mathscr{D}+\Gamma)+\omega \mathscr{U}\} \\
\times\left(\bar{U}_{s}^{(0)}-\underline{U}_{s}^{(0)}\right) \\
+\omega\left[\Gamma\left(\bar{U}_{s}^{(0)}-\underline{U}_{s}^{(0)}\right)+F\left(\bar{U}_{s}^{(0)}\right)\right. \\
\left.-F\left(\underline{U}_{s}^{(0)}\right)\right] .
\end{gathered}
$$

We have from Lemma $6, \bar{U}_{s}^{(0)} \geq \underline{U}_{s}^{(0)}$, and the nonnegative property of $[(1-\omega)(\mathscr{D}+\Gamma)+\omega \mathscr{U}]$ that $(\mathscr{D}+\Gamma-\omega \mathscr{L}) W^{(1)} \geq$ 0 . It follows from Lemma 8 again that $W^{(1)} \geq 0$. The above conclusions imply that

$$
\underline{U}_{s}^{(0)} \leq \underline{U}_{s}^{(1)} \leq \bar{U}_{s}^{(1)} \leq \bar{U}_{s}^{(0)} .
$$

We finally assume that $\underline{U}_{s}^{(m-1)} \leq \underline{U}_{s}^{(m)} \leq \bar{U}_{s}^{(m)} \leq \bar{U}_{s}^{(m-1)}$ for some $m>1$. Let $W^{(m)}=\bar{U}_{s}^{(m)}-\bar{U}_{s}^{(m+1)}$, and by (18) we have

$$
\begin{aligned}
(\mathscr{D}+\Gamma-\omega \mathscr{L}) W^{(m)}= & \{(1-\omega)(\mathscr{D}+\Gamma)+\omega \mathscr{U}\} \\
& \times\left(\bar{U}_{s}^{(m-1)}-\bar{U}_{s}^{(m)}\right) \\
& +\omega\left[\Gamma\left(\bar{U}_{s}^{(m-1)}-\bar{U}_{s}^{(m)}\right)\right. \\
& \left.+F\left(\bar{U}_{s}^{(m-1)}\right)-F\left(\bar{U}_{s}^{(m)}\right)\right] .
\end{aligned}
$$

Since $\bar{U}_{s}^{(m)} \leq \bar{U}_{s}^{(m-1)}$, we have $(\mathscr{D}+\Gamma-\omega \mathscr{L}) W^{(m)} \geq 0$. So $W^{(m)} \geq 0$ which shows that $\bar{U}_{s}^{(m+1)} \leq \bar{U}_{s}^{(m)}$. Similarly, let $V^{(m)}=\underline{U}_{s}^{(m)}-\underline{U}_{s}^{(m+1)}$ and $W^{(m+1)}=\bar{U}_{s}^{(m+1)}-\underline{U}_{s}^{(m+1)}$ simultaneously. We see that

$$
\begin{aligned}
(\mathscr{D}+\Gamma-\omega \mathscr{L}) V^{(m)}= & \{(1-\omega)(\mathscr{D}+\Gamma)+\omega \mathscr{U}\} \\
& \times\left(\underline{U}_{s}^{(m-1)}-\underline{U}_{s}^{(m)}\right) \\
& +\omega\left[\Gamma\left(\underline{U}_{s}^{(m-1)}-\underline{U}_{s}^{(m)}\right)\right. \\
& \left.+F\left(\underline{U}_{s}^{(m-1)}\right)-F\left(\underline{U}_{s}^{(m)}\right)\right] \\
\leq & 0, \quad \\
(\mathscr{D}+\Gamma-\omega \mathscr{L}) W^{(m+1)}= & \{(1-\omega)(\mathscr{D}+\Gamma)+\omega \mathscr{U}\} \\
& \times\left(\bar{U}_{s}^{(m)}-\underline{U}_{s}^{(m)}\right) \\
& +\omega\left[\Gamma\left(\bar{U}_{s}^{(m)}-\underline{U}_{s}^{(m)}\right)\right. \\
& \left.+F\left(\bar{U}_{s}^{(m)}\right)-F\left(\underline{U}_{s}^{(m)}\right)\right]
\end{aligned}
$$

$\geq 0$. 
By $(\mathscr{D}+\Gamma-\omega \mathscr{L})^{-1} \geq 0$, we have $V^{(m)} \leq 0$, and $W^{(m+1)} \geq 0$. Hence, $\underline{U}_{s}^{(m)} \leq \underline{U}_{s}^{(m+1)}$, and $\bar{U}_{s}^{(m+1)} \geq \underline{U}_{s}^{(m+1)}$. The proof of monotone property (46) is completed.

To show that $\bar{U}_{s}^{(m)}$ is an upper solution for each $m$, we observe from (18) that

$$
\begin{aligned}
(\mathscr{D}+\Gamma-\omega \mathscr{L}) \bar{U}_{s}^{(m)}= & \left\{[(1-\omega)(\mathscr{D}+\Gamma)+\omega \mathcal{U}] \bar{U}_{s}^{(m-1)}\right. \\
& \left.+\omega\left[\Gamma \bar{U}_{s}^{(m-1)}+F\left(\bar{U}_{s}^{(m-1)}\right)+G^{*}\right]\right\}, \\
\omega \mathscr{D} \bar{U}_{s}^{(m)}-\omega \mathscr{L} \bar{U}_{s}^{(m)}=\omega \mathscr{D} \bar{U}_{s}^{(m)}-\mathscr{D} \bar{U}_{s}^{(m)} & +\mathscr{D} \bar{U}_{s}^{(m-1)}-\omega \mathscr{D} \bar{U}_{s}^{(m-1)} \\
& +\Gamma \bar{U}_{s}^{(m-1)}-\Gamma \bar{U}_{s}^{(m)}+\omega \mathscr{U} \bar{U}_{s}^{(m-1)} \\
& +\omega F\left(\bar{U}_{s}^{(m-1)}\right)+\omega G^{*}, \\
\bar{D}_{s}^{(m)}-\omega \mathscr{L} \bar{U}_{s}^{(m)}= & (1-\omega) \mathscr{D}\left(\bar{U}_{s}^{(m-1)}-\bar{U}_{s}^{(m)}\right) \\
& +\omega \mathscr{U} \bar{U}_{s}^{(m-1)}+\Gamma\left(\bar{U}_{s}^{(m-1)}-\bar{U}_{s}^{(m)}\right) \\
& +\omega F\left(\bar{U}_{s}^{(m-1)}\right)+\omega G^{*} .
\end{aligned}
$$

By $0<\omega<1$ and (46), we obtain

$$
\begin{aligned}
& \omega \mathscr{D} \bar{U}_{s}^{(m)}-\omega \mathscr{L} \bar{U}_{s}^{(m)} \geq \omega \mathcal{U} \bar{U}_{s}^{(m)}+\omega \Gamma\left(\bar{U}_{s}^{(m-1)}-\bar{U}_{s}^{(m)}\right) \\
& +\omega F\left(\bar{U}_{s}^{(m-1)}\right)+\omega G^{*} \\
& \omega(\mathscr{D}-\mathscr{L}-\mathscr{U}) \bar{U}_{s}^{(m)} \geq \omega\left[\Gamma\left(\bar{U}_{s}^{(m-1)}-\bar{U}_{s}^{(m)}\right)\right. \\
& \left.+F\left(\bar{U}_{s}^{(m-1)}\right)+G^{*}\right] \\
& A \bar{U}_{s}^{(m)} \geq \Gamma\left(\bar{U}_{s}^{(m-1)}-\bar{U}_{s}^{(m)}\right)+F\left(\bar{U}_{s}^{(m-1)}\right)+G^{*} .
\end{aligned}
$$

By Lemma 6, we have

$$
A \bar{U}_{s}^{(m)} \geq F\left(\bar{U}_{s}^{(m)}\right)+G^{*}
$$

This shows that $\bar{U}_{s}^{(m)}$ is an upper solution for each $m$. Similarly, we have

$$
A \underline{U}_{s}^{(m)} \leq F\left(\underline{U}_{s}^{(m)}\right)+G^{*}
$$

that is, for each $m, \underline{U}_{s}^{(m)}$ is a lower solution, and thus the proof is completed.
Theorem 16. Let $\widetilde{U}, \widehat{U}$ be a pair of ordered upper and lower solutions of (10). Then the sequences $\left\{\bar{U}_{s}^{(m)}\right\}_{m=0}^{\infty},\left\{\underline{U}_{s}^{(m)}\right\}_{m=0}^{\infty}$ given by (18) with $\bar{U}_{s}^{(0)}=\widetilde{U}$ and $\underline{U}_{s}^{(0)}=\widehat{U}$ converge monotonically to solutions $\bar{U}_{s}$ and $\underline{U}_{s}$ of (10), respectively, where

$$
\begin{aligned}
& \bar{U}_{s} \stackrel{\text { def }}{=}\left(\bar{u}_{00}, \bar{u}_{10}, \ldots, \bar{u}_{n 0}, \ldots, \bar{u}_{0 m}, \bar{u}_{1 m}, \ldots, \bar{u}_{n m}\right)^{T}, \\
& \underline{U}_{s} \stackrel{\text { def }}{=}\left(\underline{u}_{00}, \underline{u}_{10}, \ldots, \underline{u}_{n 0}, \ldots, \underline{u}_{0 m}, \underline{u}_{1 m}, \ldots, \underline{u}_{n m}\right)^{T} .
\end{aligned}
$$

Moreover,

$$
\begin{array}{r}
\widehat{U} \leq \underline{U}_{s}^{(m)} \leq \underline{U}_{s}^{(m+1)} \leq \underline{U}_{s} \leq \bar{U}_{s} \leq \bar{U}_{s}^{(m+1)} \leq \bar{U}_{s}^{(m)} \leq \widetilde{U} \\
m=1,2, \ldots,
\end{array}
$$

and if $U^{*}$ is any solution in $\langle\widehat{U}, \widetilde{U}\rangle$, then $\underline{U}_{s} \leq U^{*} \leq \bar{U}_{s}$.

Proof. By Theorem 15, the limits $\lim \bar{U}^{(m)}=\bar{U}_{s}$ and $\lim \underline{U}^{(m)}=\underline{U}_{s}$ as $m \rightarrow \infty$ exist, and the relation (58) also holds. Letting $m \rightarrow \infty$ in (18) shows that $\bar{U}_{s}$ and $\underline{U}_{s}$ are solutions of (15). The equivalence between (10) and (15) ensures that $\bar{U}_{s}$ and $\underline{U}_{s}$ are solutions of (10). Now if $U^{*}$ is a solution in $\langle\widehat{U}, \widetilde{U}\rangle$, then $\widetilde{U}$ and $U^{*}$ are ordered upper and lower solutions. Using $\bar{U}_{s}^{(0)}=\widetilde{U}$ and $\underline{U}_{s}^{(0)}=U^{*}$, Theorem 15 implies that $\bar{U}_{s}^{(m)} \geq U^{*}$ for every $m$. Letting $m \rightarrow \infty$ gives $\bar{U}_{s} \geq U^{*}$. A similar argument using $U^{*}$ and $\widehat{U}$ as ordered upper and lower solutions yields $U^{*} \geq \underline{U}_{S}$. This proves the theorem.

In Theorem 16, $\bar{U}_{s}$ and $\underline{U}_{s}$ are often called maximal and minimal solutions in $\langle\widehat{U}, \widetilde{U}\rangle$, respectively. In general, these two solutions are not necessarily the same. Let $A$ be symmetric. Then $A$ has real and positive eigenvalues [2]. However, if $\sigma<\mu$, where $\underline{\mu}$ is the smallest positive eigenvalue of $A$ and

$$
\sigma \equiv \max \left\{\frac{\partial f_{i j}}{\partial u}\left(u_{i j}\right): \widehat{u}_{i j} \leq u_{i j} \leq \tilde{u}_{i j},(i, j) \in \bar{\Upsilon}\right\}
$$

then the following theorem holds.

Theorem 17. Let the conditions in Theorem 16 hold. If either $\sigma \leq 0$ or $\sigma<\mu$ and $A$ is symmetric, then $\bar{U}_{s}=\underline{U}_{s}$ and is the unique solution of (10).

Proof. Let $W=\bar{U}_{s}-\underline{U}_{s}$ and $\bar{u}_{i j}$ and $\underline{u}_{i j}$ be the components of $\bar{U}_{s}$ and $\underline{U}_{s}$, respectively. By (58), we have $W \geq 0$. On the other hand, by the mean value theorem, we have

$$
\frac{f\left(x_{i}, y_{j}, \bar{u}_{i j}\right)-f\left(x_{i}, y_{j}, \underline{u}_{i j}\right)}{\bar{u}_{i j}-\underline{u}_{i j}}=\frac{\partial f_{i j}}{\partial u}\left(u_{i j}\right) \leq \sigma,
$$


where $u_{i j}$ lies between $\underline{u}_{i j}$ and $\bar{u}_{i j}$. Hence,

$$
\begin{aligned}
A W & =A\left(\bar{U}_{s}-\underline{U}_{s}\right)=A \bar{U}_{s}-A \underline{U}_{s} \\
& =F\left(\bar{U}_{s}\right)+G^{*}-F\left(\underline{U}_{s}\right)-G^{*} \\
& =F\left(\bar{U}_{s}\right)-F\left(\underline{U}_{s}\right) \\
& \leq \sigma\left(\bar{U}_{s}-\underline{U}_{s}\right) \\
& =\sigma W,
\end{aligned}
$$

and thus

$$
(A-\sigma I) W \leq 0
$$

Case 1. If $\sigma \leq 0$. From the form of $A,(A-\sigma I)$ is strictly diagonally dominant. It follows that $(A-\sigma I)^{-1} \geq 0$, and thus $W \leq 0$.

Case 2. If $\sigma<\mu$. Since $\mu$ is the smallest positive eigenvalue of $A$, then $\mu^{-1}$ is the biggest positive eigenvalue of $A^{-1}$. From Theorem 7 and $A^{-1} \geq 0$, we have

$$
\begin{gathered}
\sigma^{-1}>\underline{\mu}^{-1} \geq \rho\left(A^{-1}\right), \\
\left(\sigma^{-1} I-A^{-1}\right)^{-1} \geq 0, \\
\sigma^{-1} A^{-1}\left(\sigma^{-1} I-A^{-1}\right)^{-1} \geq 0, \\
(A-\sigma I)^{-1} \geq 0 .
\end{gathered}
$$

Hence, $W \leq 0$. So we know that $W=0$. This proves $\bar{U}_{s}=\underline{U}_{s}$. The uniqueness follows from the relation $\bar{U}_{s} \geq U^{*} \geq \underline{U}_{s}$ for any solution $U^{*} \in\langle\widehat{U}, \widetilde{U}\rangle$.

Remark 18. For system (1), the well-known method of upper and lower solutions with SORMI is applied for the case $0<$ $\omega<1$ (see Theorems 15, 16, and 17). However, the nonnegative property of $(1-\omega)(\mathscr{D}+\Gamma)+\omega \mathscr{U}$ is not available when $\omega>1$. A new approach for solving this problem by the boundedness of the SORMI sequences and Cauchy sequence property is proposed. To make sure of the convergence of the SORMI sequences, the assumptions ( $\mathrm{H} 1),(\mathrm{H} 2)$, and (H3) are necessary. But it should be pointed out that these constraints are not easy to be verified. It is important to weaken these constraints when the SORMI method is applied to realistic problems. Fixed point theory is a powerful tool to overcome this problem for further study.

\section{Numerical Results}

Assume that the matrix $A$ of (10) is an $n \times n$ matrix. The componentwise SORMI algorithm is given as follows:

$$
\begin{gathered}
u_{i}^{(m+1)}=\omega\left[\left(\sum_{j=1}^{i-1} a_{i j} u_{j}^{(m+1)}+\sum_{j=i+1}^{n} a_{i j} u_{j}^{(m)}\right.\right. \\
\left.\left.+\bar{\gamma}_{i} u_{i}^{(m)}+h k f_{i}\left(u_{i}^{(m)}\right)+g_{i}^{*}\right)\left(a_{i i}+\bar{\gamma}_{i}\right)^{-1}\right] \\
+(1-\omega) u_{i}^{(m)}, \quad i=1, \ldots, n .
\end{gathered}
$$

Another equivalent form is

$$
\begin{aligned}
u_{i}^{(m+1)}=\omega & {\left[\left(\sum_{j=1}^{i-1} a_{i j} u_{j}^{(m+1)}+\sum_{j=i+1}^{n} a_{i j} u_{j}^{(m)}\right.\right.} \\
& \left.\left.+h k f_{i}\left(u_{i}^{(m)}\right)+g_{i}^{*}\right)\left(a_{i i}+\bar{\gamma}_{i}\right)^{-1}\right] \\
+ & \left(1-\frac{\omega a_{i i}}{a_{i i}+\bar{\gamma}_{i}}\right) u_{i}^{(m)}, \quad i=1, \ldots, n .
\end{aligned}
$$

The main requirement for the application of the various MI schemes is the existence of a pair of ordered upper and lower solutions. To ensure the existence, the nonlinear function $f$ must have some necessary conditions. Hence, in Section 1, we require that $f_{i j}\left(u_{i j}\right)$ is uniformly bounded in $\mathfrak{R}$. Now we present some numerical results with two test problems.

Example 19. Consider the one-dimensional boundary value problem:

$$
\begin{array}{r}
-u^{\prime \prime}=-\frac{1}{2} u+\frac{5}{2} \pi \sin (\sqrt{2} x)-\frac{1}{2} x \sin (\sqrt{2} \pi), \\
0<x<\pi, \quad u(0)=u(\pi)=0 .
\end{array}
$$

The exact solution is

$$
u(x)=\pi \sin (\sqrt{2} x)-x \sin (\sqrt{2} \pi) .
$$

Let $f(x, u)=(-1 / 2) u+(5 / 2) \pi \sin (\sqrt{2} x)-(1 / 2) x \sin (\sqrt{2} \pi)$, and choose $\Gamma=\operatorname{diag}(2,2, \ldots, 2), U_{s}^{(0)}=(0,0, \ldots, 0)^{T}$. Then,

$$
\begin{aligned}
\left|f\left(x, u_{1}\right)-f\left(x, u_{2}\right)\right| & =\frac{1}{2}\left|u_{1}-u_{2}\right| \\
& \stackrel{\text { def }}{=} \mathscr{H}(c)\left|u_{1}-u_{2}\right| .
\end{aligned}
$$

Hence we choose $\lambda=1 / 2$. We examine the assumptions (H3) and (H4) and the convergence of the SORMI sequences. The numerical results are given in Table 1, and the exact and approximate solutions are shown in Figure 1. Moreover, we sketch the relation between the numbers of iterations and relaxation factors $\omega$ by Figure 2 . 
TABLE 1: Numbers of iterations and $\eta$ values for Example 19.

\begin{tabular}{|c|c|c|c|c|c|c|}
\hline$\omega$ & 0.2 & 0.25 & 0.3 & 0.4 & 0.5 & 0.6 \\
\hline Number of iterations & 18330 & 14745 & 12268 & 9046 & 7024 & 5626 \\
\hline$\|B\|$ & 0.9999 & 0.9998 & 0.9998 & 0.9997 & 0.9997 & 0.9996 \\
\hline$\eta$ & 0.9999 & 0.9999 & 0.9999 & 0.9999 & 0.9998 & 0.9998 \\
\hline$\omega$ & 0.8 & 1.0 & 1.2 & 1.6 & 1.8 & 1.9 \\
\hline Number of iterations & 3800 & 2645 & 1837 & 751 & 342 & 127 \\
\hline$\|B\|$ & 0.9994 & 0.9992 & 0.9990 & 0.9985 & 0.9982 & 0.9980 \\
\hline$\eta$ & 0.9997 & 0.9996 & 0.9995 & 0.9993 & 0.9992 & 0.9991 \\
\hline$\omega$ & 1.92 & 1.95 & 1.96 & 1.97 & 1.98 & 1.99 \\
\hline Number of iterations & 151 & 236 & 295 & 381 & 535 & 940 \\
\hline$\|B\|$ & 0.9979 & 0.9979 & 0.9979 & 0.9978 & 0.9978 & 0.9978 \\
\hline$\eta$ & 0.9991 & 0.9990 & 0.9990 & 0.9990 & 0.9990 & 0.9990 \\
\hline
\end{tabular}

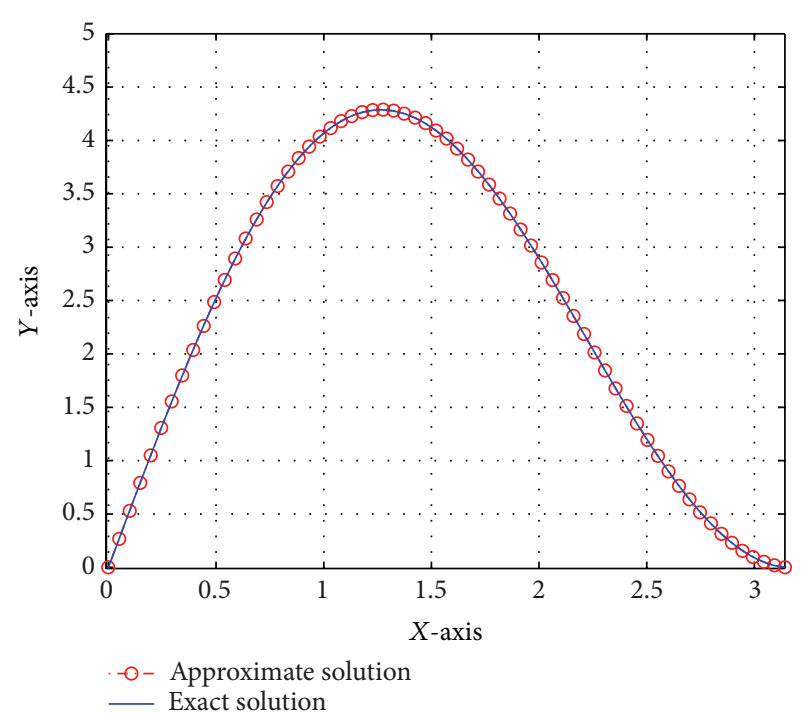

FIgURE 1: The exact and approximate solutions for Example 19.

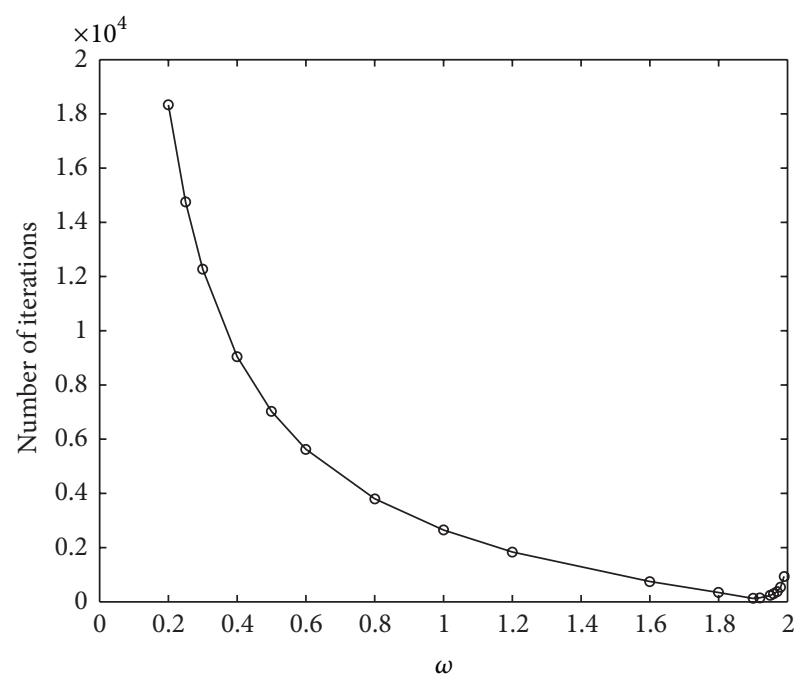

Figure 2: Numbers of iterations and $\omega$ values for Example 19.
The numbers of iterations for the SORMI method are listed in Table 1. We focus on the SORMI method with $\omega=$ 1.9. The number of iterations is 127 . Compared with it, the Jacobi and Gauss-Seidel MI methods require 4905 and 2645 iterations, respectively. The resulting increase in the speed of convergence is very dramatic. Moreover, the values of $\|B\|$ and $\eta$ in Table 1 are smaller than 1 which verify our theory of the boundedness and convergence for the SORMI method.

Example 20. As the second test problem, consider the logistic model in population growth problem with nonlinear function

$$
f(x, y, u)=\sigma u(1-u)+q(x, y)
$$

where $\sigma$ is a positive constant, and $q(x, y)$ is a possible internal source [6]. The discretized function is given by

$$
f_{i j}\left(u_{i j}\right)=\sigma u_{i j}\left(1-u_{i j}\right)+q_{i j}
$$

For physical reasons, we suppose that $f(x, y, u) \geq 0, q(x, y) \geq$ 0 , and $g(x, y) \geq 0$, such that $\widehat{U}=\mathbf{0}$ is a lower solution of (10). For upper solution, consider the following.

Case 1. $q(x, y)>0$ and $g(x, y)>0$. (i) If the upper solution is dependent on $\sigma$, define $K^{(1)}=\sigma / 4+\bar{q}+\bar{g}^{*}, V=$ $\left(K^{(1)}, K^{(1)}, \ldots, K^{(1)}\right)$, and $A \widetilde{U}=V$. Then $\widetilde{U}$ is an upper solution, where $\bar{q}$ and $\bar{g}^{*}$ are any upper bounds of $q_{i, j}$ and $g_{i, j}^{*}$, respectively. (ii) If upper solution is independent of $\sigma$, then $\widetilde{U}=P+V^{*}$ is an upper solution, where $P \stackrel{\text { def }}{=}(1,1, \ldots, 1)^{T}$, and $Q$ is the vector with components $q_{i, j}$ which satisfies $Q+G^{*}=$ $A V^{*}$.

Case 2. (i) If $g=0$, then $\widetilde{U}=(K, K, \ldots, K)^{T}$ is an upper solution, where $K$ is a constant which satisfies $\sigma K(K-1) \geq \bar{q}$. (ii) If $q=g=0$, then $\widetilde{U}=P$ is an upper solution. 
We now give a model problem where the exact solution is known explicitly [6]. This problem is given by

$$
\begin{gathered}
-\left(u_{x x}+u_{y y}\right)=\sigma u(1-u)+q(x, y), \\
0<x<1,0<y<2, \\
u_{x}(0, y)=u(1, y)=u(x, 0)=u(x, 2)=0 .
\end{gathered}
$$

It is easy to verify that when $\sigma=\pi^{2} / 4$ and

$$
q(x, y)=2 \sin \left(\frac{\pi y}{2}\right)+\frac{\pi^{2}}{4}\left(1-x^{2}\right)^{2} \sin ^{2}\left(\frac{\pi y}{2}\right),
$$

the exact solution of (71) is given by

$$
u(x, y)=\left(1-x^{2}\right) \sin \left(\frac{\pi y}{2}\right)
$$

Since $0 \leq q(x, y) \leq 2+\pi^{2} / 4$ for $(x, y) \in[0,1] \times[0,2]$, the constant pair $\widetilde{U}=(K, K, \ldots, K)^{T}$ and $\widehat{U}=\mathbf{0}$ are ordered upper and lower solutions whenever $\left(\pi^{2} / 4\right) K(K-1) \geq 2+$ $\pi^{2} / 4$ with $K=2$. Hence, (71) is case 2 whenever $g=0$, and we have the upper and lower solutions $\widehat{U}=(0,0, \ldots, 0)^{T}$ and $\widetilde{U}=(2,2, \ldots, 2)^{T}$. Let $f(x, y, u)=\left(\pi^{2} / 4\right) u(1-u)+q(x, y)$. We define

$$
\gamma=\max _{0 \leq u \leq 2}\left|\frac{\partial f(x, y, u)}{\partial u}\right|=\frac{3 \pi^{2}}{4}, \quad \Gamma=\operatorname{diag}(\gamma, \gamma, \ldots, \gamma)^{T} .
$$

If $0 \leq u_{1}, u_{2} \leq c$, then

$$
\begin{aligned}
\left|f\left(x, y, u_{1}\right)-f\left(x, y, u_{2}\right)\right|= & \mid \sigma u_{1}\left(1-u_{1}\right)+q(x, y) \\
& -\sigma u_{2}\left(1-u_{2}\right)-q(x, y) \mid \\
= & \left|\sigma u_{1}-\sigma u_{1}^{2}-\sigma u_{2}+\sigma u_{2}^{2}\right| \\
= & \left|\sigma\left(u_{2}^{2}-u_{1}^{2}\right)-\sigma\left(u_{2}-u_{1}\right)\right| \\
= & \mid \sigma\left(u_{2}+u_{1}\right)\left(u_{2}-u_{1}\right) \\
& -\sigma\left(u_{2}-u_{1}\right) \mid \\
= & \left|\sigma\left(u_{2}+u_{1}-1\right)\left(u_{2}-u_{1}\right)\right| \\
= & \sigma\left|u_{2}+u_{1}-1\right|\left|u_{1}-u_{2}\right| \\
\leq & \sigma|2 c-1|\left|u_{1}-u_{2}\right| \\
\text { def } & \mathscr{H}(c)\left|u_{1}-u_{2}\right| .
\end{aligned}
$$

The SORMI method takes 94 iterations with $\omega=1.92$ when $U^{(0)}=0$ is applied. The approximate solution is similar to the exact solution as shown in Figure 3. Since they are similar, we omit to sketch the exact solution figure. In comparison with the SORMI method, the Jacobi and Gauss-Seidel MI methods require 2243 and 1249 iterations, respectively. The reduction of iterations is significant. The numerical results are listed in
TABLE 2: Numbers of iterations and $\eta$ values for Example 20.

\begin{tabular}{lcccc}
\hline$\omega$ & 0.05 & 0.1 & 0.2 & 0.3 \\
\hline Number of iterations & 28503 & 15706 & 8344 & 5612 \\
$\|B\|$ & 0.9999 & 0.9997 & 0.9995 & 0.9992 \\
$\eta$ & 0.9999 & 0.9998 & 0.9996 & 0.9994 \\
\hline$\omega$ & 0.4 & 0.5 & 0.8 & 1.0 \\
\hline Number of iterations & 4155 & 3239 & 1775 & 1249 \\
$\|B\|$ & 0.9989 & 0.9986 & 0.9976 & 0.9969 \\
$\eta$ & 0.9992 & 0.9989 & 0.9982 & 0.9976 \\
\hline$\omega$ & 1.2 & 1.6 & 1.8 & 1.9 \\
\hline Number of iterations & 881 & 386 & 200 & 104 \\
$\|B\|$ & 0.9961 & 0.9943 & 0.9933 & 0.9928 \\
$\eta$ & 0.9970 & 0.9955 & 0.9947 & 0.9943 \\
\hline$\omega$ & 1.92 & 1.95 & 1.97 & 1.99 \\
\hline Number of iterations & 94 & 129 & 185 & 307 \\
$\|B\|$ & 0.9927 & 0.9925 & 0.9924 & 0.9923 \\
$\eta$ & 0.9942 & 0.9942 & 0.9942 & 0.9944 \\
\hline
\end{tabular}

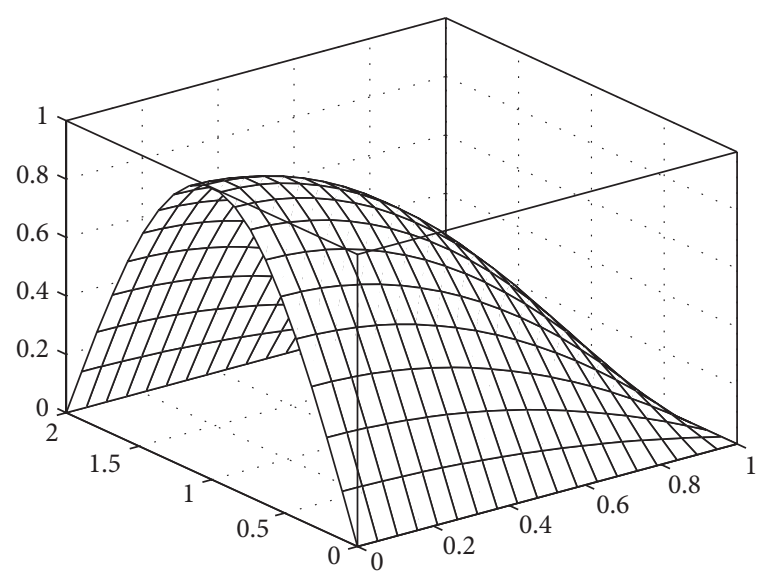

FIGURE 3: The approximate solution for Example 20.

Table 2. The relation between the numbers of iterations and relaxation factors $\omega$ is shown in Figure 4. They verify our theory of the boundedness and convergence for the SORMI method again.

\section{Conclusions}

A new iterative method for solving nonlinear equations is developed in this paper. It combines SOR method with MI scheme and therefore gives rise to the terminology "SORMI." The boundedness and convergence of the SORMI sequence are proven under some suitable assumptions. Some numerical examples are given to verify our theory of the boundedness and convergence for the SORMI method. Moreover, the reduction of iterations is quite significant in comparison with the Jacobi and Gauss-Seidel MI methods. 


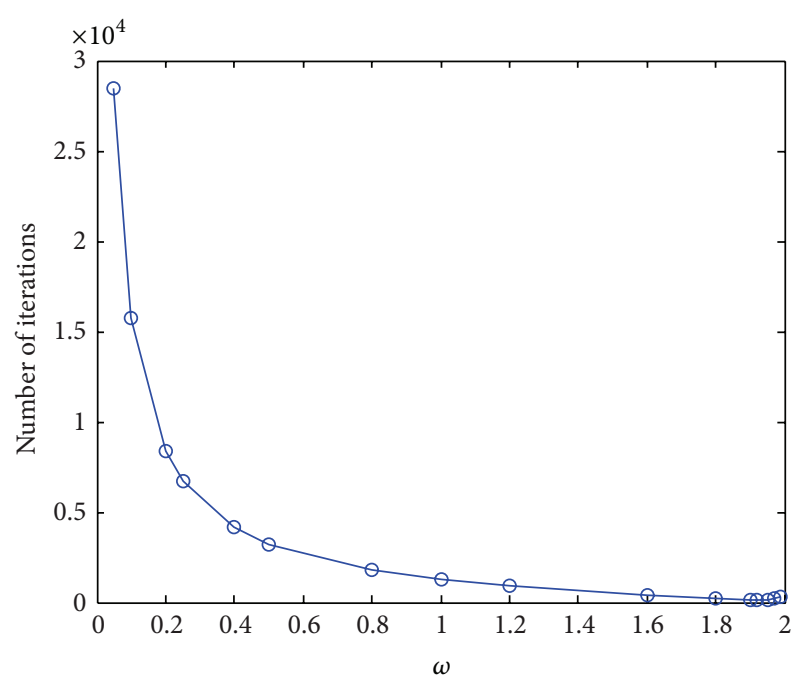

FIgURE 4: Numbers of iterations and $\omega$ values for Example 20.

\section{Acknowledgments}

This work was supported by the National Science Council of Taiwan under the Grants 100-2115-M-017-004-MY2. The authors would like to thank the referees for their valuable comments and suggestions which improved the presentation of the paper.

\section{References}

[1] A. Berman and R. J. Plemmons, Nonnegative Matrices in the Mathematical Sciences, Academic Press, New York, NY, USA, 1979.

[2] R. S. Varga, Matrix Iterative Analysis, Prentice-Hall, Englewood Cliffs, NJ, USA, 1962.

[3] D. M. Young, Iterative Solution of Large Linear Systems, Academic Press, New York, NY, USA, 1971.

[4] R. P. Agarwal and Y. M. Wang, "Some recent developments of Numerov's method," Computers \& Mathematics with Applications, vol. 42, no. 3-5, pp. 561-592, 2001.

[5] D. Greenspan and S. V. Parter, "Mildly nonlinear elliptic partial differential equations and their numerical solution. II," Numerische Mathematik, vol. 7, pp. 129-146, 1965.

[6] C. V. Pao, "Block monotone iterative methods for numerical solutions of nonlinear elliptic equations," Numerische Mathematik, vol. 72, no. 2, pp. 239-262, 1995.

[7] C. V. Pao, "Numerical methods for quasi-linear elliptic equations with nonlinear boundary conditions," SIAM Journal on Numerical Analysis, vol. 45, no. 3, pp. 1081-1106, 2007.

[8] S. V. Parter, "Mildly nonlinear elliptic partial differential equations and their numerical solution. I," Numerische Mathematik, vol. 7, pp. 113-128, 1965.

[9] S. Heikkilä and V. Lakshmikantham, Monotone Iterative Techniques for Discontinuous Nonlinear Differential Equations, Marcel Dekker, New York, NY, USA, 1994.

[10] C. V. Pao, Nonlinear Parabolic and Elliptic Equations, Plenum Press, New York, NY, USA, 1992.
[11] C. V. Pao and X. Lu, "Block monotone iterative method for semilinear parabolic equations with nonlinear boundary conditions," SIAM Journal on Numerical Analysis, vol. 47, no. 6, pp. 4581-4606, 2010.

[12] Y. M. Wang, "A modified accelerated monotone iterative method for finite difference reaction-diffusion-convection equations," Journal of Computational and Applied Mathematics, vol. 235, no. 12, pp. 3646-3660, 2011.

[13] R. P. Agarwal, Difference Equations and Inequalities, Marcel Dekker, New York, NY, USA, 1992.

[14] R. Kannan and M. B. Ray, "Monotone iterative methods for nonlinear equations involving a noninvertible linear part," Numerische Mathematik, vol. 45, no. 2, pp. 219-225, 1984.

[15] A. W. Leung and D. A. Murio, "Accelerated monotone scheme for finite difference equations concerning steady-state preypredator interactions," Journal of Computational and Applied Mathematics, vol. 16, no. 3, pp. 333-341, 1986.

[16] K. Ishihara, "Monotone explicit iterations of the finite element approximations for the nonlinear boundary value problem," Numerische Mathematik, vol. 43, no. 3, pp. 419-437, 1984.

[17] C. A. Brebbia and S. Walker, Boundary Element Techniques in Engineering, Newnes-Butterworths, London, UK, 1980.

[18] Y. Deng, G. Chen, W. M. Ni, and J. Zhou, "Boundary element monotone iteration scheme for semilinear elliptic partial differential equations," Mathematics of Computation, vol. 65, no. 215, pp. 943-982, 1996.

[19] J. M. Ortega and W. C. Rheinboldt, Iterative Solution of Nonlinear Equations in Several Variables, Academic Press, New York, NY, USA, 1970.

[20] X. Lu, "Combined iterative methods for numerical solutions of parabolic problems with time delays," Applied Mathematics and Computation, vol. 89, no. 1-3, pp. 213-224, 1998.

[21] R. C. Chen and J. L. Liu, "Monotone iterative methods for the adaptive finite element solution of semiconductor equations," Journal of Computational and Applied Mathematics, vol. 159, no. 2, pp. 341-364, 2003.

[22] R. C. Chen and J. L. Liu, "An iterative method for adaptive finite element solutions of an energy transport model of semiconductor devices," Journal of Computational Physics, vol. 189, no. 2, pp. 579-606, 2003.

[23] R. C. Chen and J. L. Liu, "A quantum corrected energy-transport model for nanoscale semiconductor devices," Journal of Computational Physics, vol. 204, no. 1, pp. 131-156, 2005.

[24] R. C. Chen, "An iterative method for finite-element solutions of the nonlinear Poisson-Boltzmann equation," WSEAS Transactions on Computers, vol. 7, no. 4, pp. 165-173, 2008.

[25] W. F. Ames, Numerical Methods for Partial Differential Equations, Academic Press, Boston, Mass, USA, 1992.

[26] A. A. Samarskii, The Theory of Difference Schemes, vol. 240, Marcel Dekker, New York, NY, USA, 2001.

[27] K. W. Morton, Numerical Solution of Convection-Diffusion Problems, vol. 12, Chapman \& Hall, London, UK, 1996.

[28] Y. Saad, Iterative Methods for Sparse Linear Systems, Society for Industrial and Applied Mathematics, Philadelphia, Pa, USA, 2nd edition, 2003. 


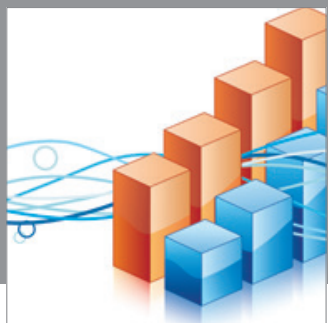

Advances in

Operations Research

mansans

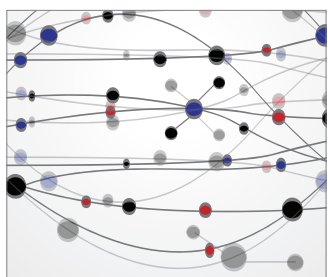

The Scientific World Journal
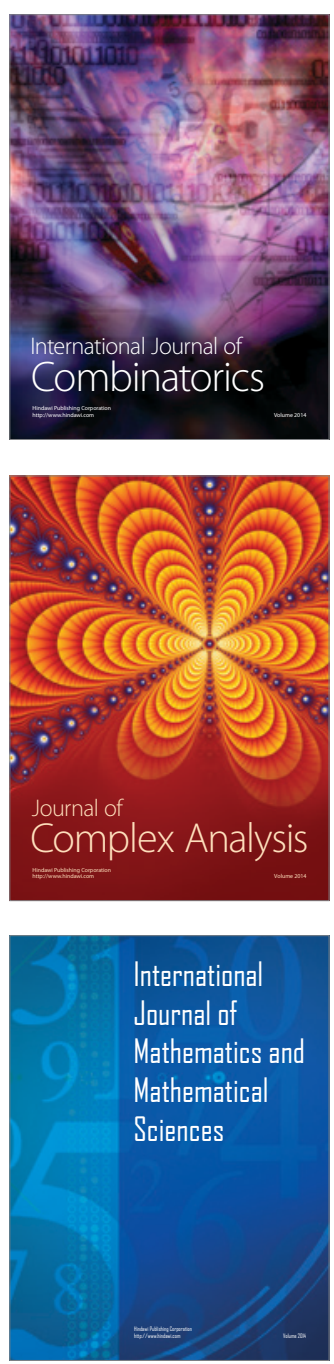
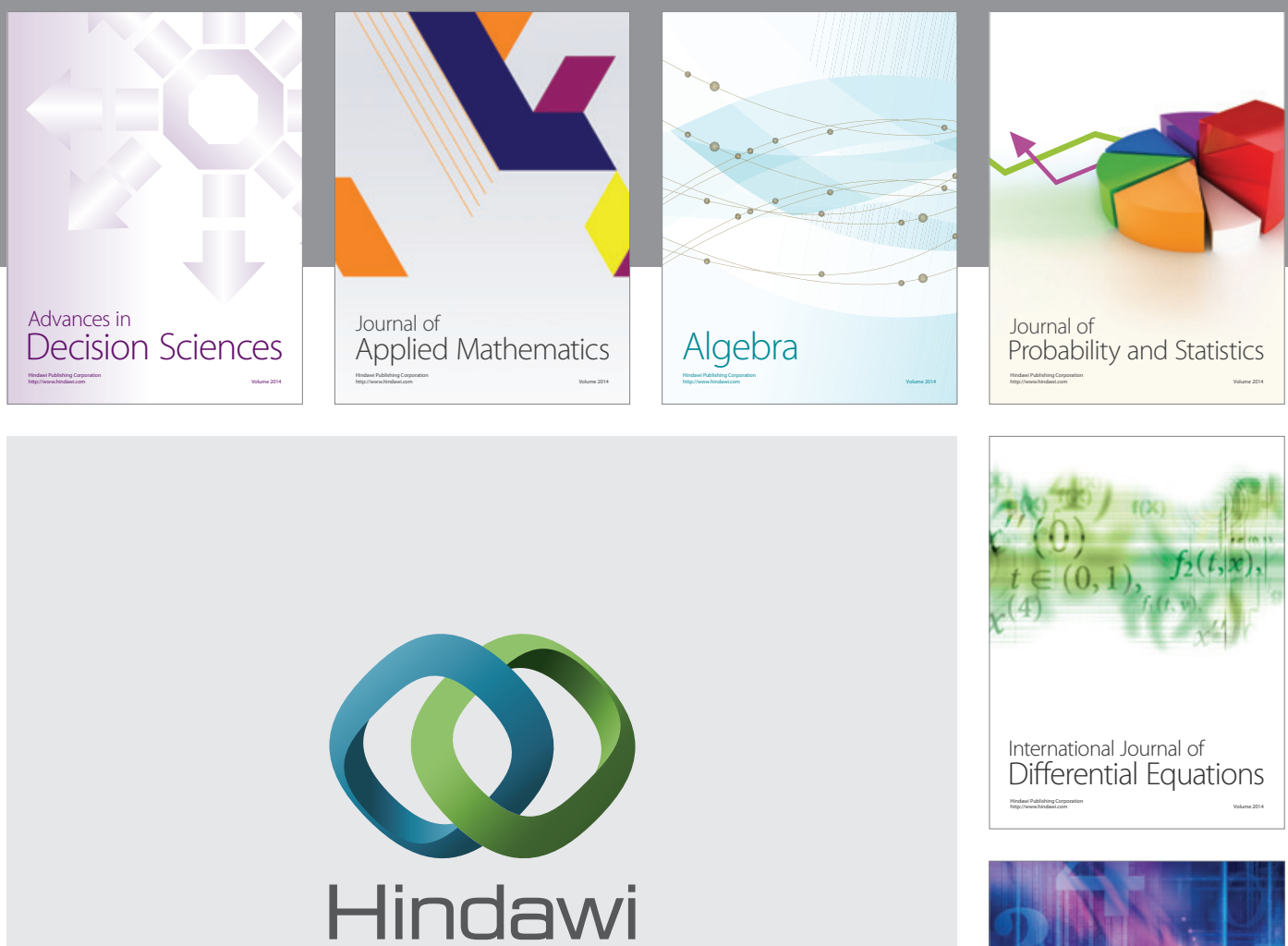

Submit your manuscripts at http://www.hindawi.com
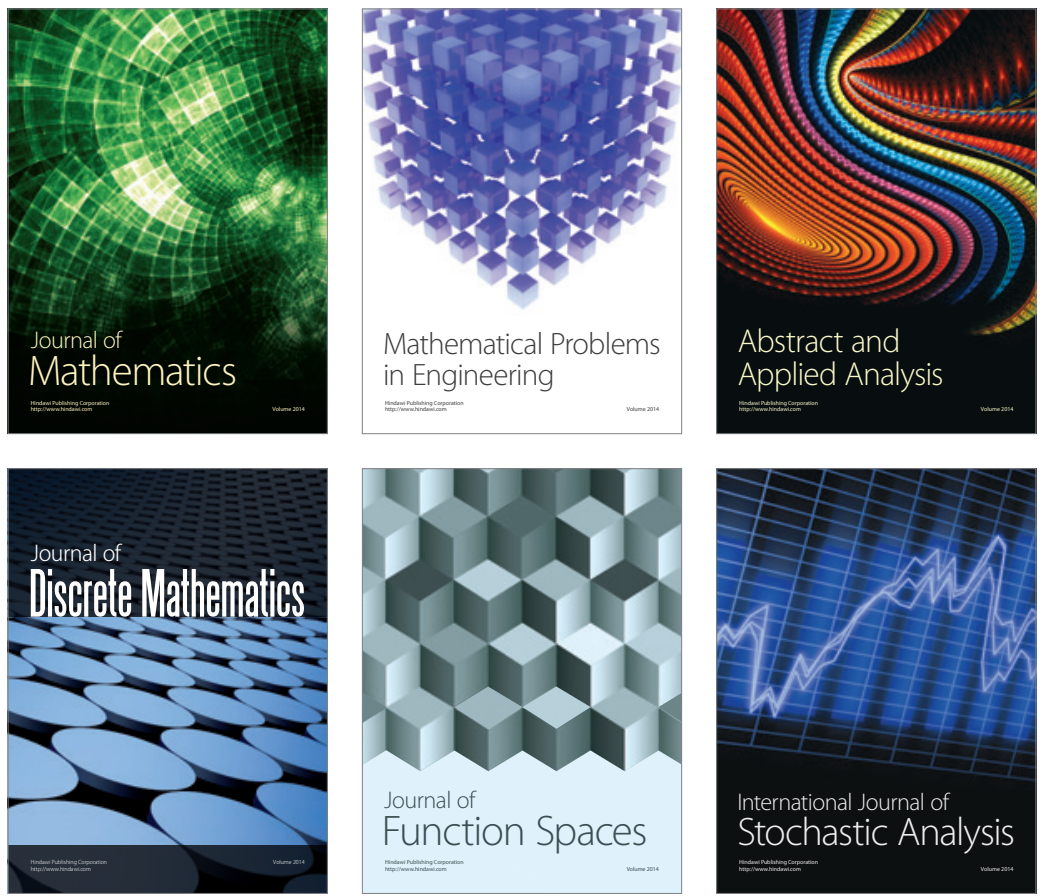

Journal of

Function Spaces

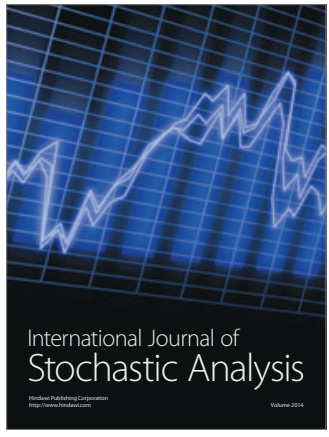

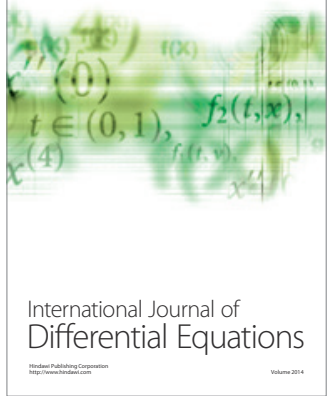
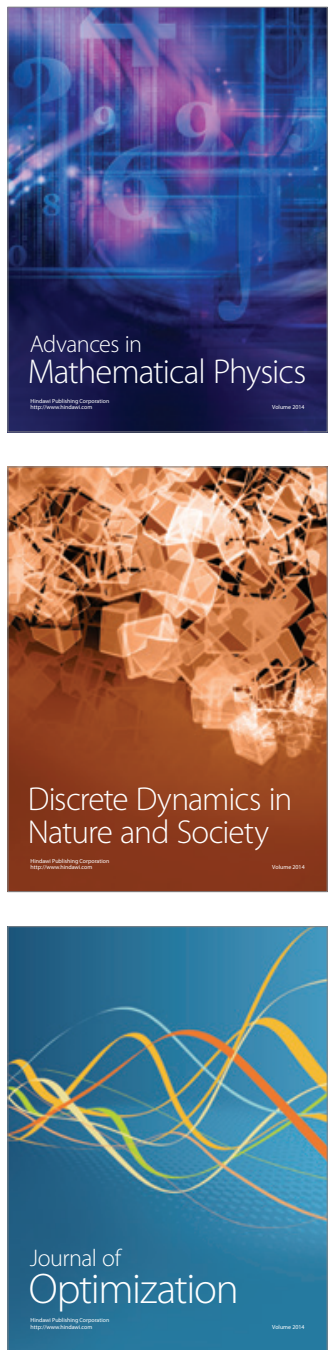